Int. J. Electrochem. Sci., 15 (2020) 493 - 504

\title{
Electrocoagulation with a nanosecond pulse power supply to remove COD from municipal wastewater using iron electrodes
}

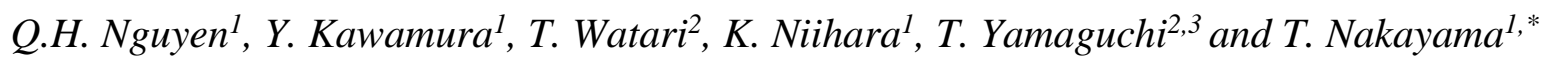 \\ ${ }^{1}$ Extreme Energy-Density Research Institute, Nagaoka University of Technology, 1603-1 \\ Kamitomioka, Nagaoka, Niigata, 940-2188, Japan \\ ${ }^{2}$ Department of Civil and Environmental Engineering, Nagaoka University of Technology, 1603-1 \\ Kamitomioka, Nagaoka, Niigata, 940-2188, Japan \\ ${ }^{3}$ Department of Science of Technology Innovation, Nagaoka University of Technology, 1603-1 \\ Kamitomioka, Nagaoka, Niigata, 940-2188, Japan \\ *E-mail: nky15@vos.nagaokaut.ac.jp
}

doi: $10.20964 / 2020.01 .66$

Received: 4 August 2019 / Accepted: 10 October 2019 / Published: 30 November 2019

\begin{abstract}
Electrocoagulation (EC) with iron electrodes was used to remove the chemical oxygen demand (COD) of municipal wastewater. A nanosecond pulse power supply (NSP) was evaluated and compared with direct current (DC). During the EC process, municipal wastewater was collected from the sewage center in Nagaoka City, Japan. Four Fe electrodes were connected in a monopolar parallel configuration (MPP). The obtained results indicated that the maximum COD removal efficiencies were $72 \%$ and $82 \%$ using DC and NSP, respectively. The use of an NSP allows EC operation at high voltage without the breakdown of the medium between the electrodes, promoting iron hydroxide flocs and the quantity of hydrogen bubbles as well as enhancing the COD removal efficiency with low specific energy consumption (SEC). At a similar COD removal efficiency $(\sim 72 \%)$, compared with DC, an NSP could reduce the SEC by approximately $40 \%$. Therefore, compared with DC, the use of an NSP allows lowcost operation and enhances the COD removal efficiency from municipal wastewater. Analysis of the electrochemically generated byproducts by field emission scanning electron microscopy (FE-SEM), Xray diffraction (XRD), and energy dispersive spectroscopy (EDS) showed that NSP flocs had higher adsorption properties for pollutants than DC flocs. The results permitted us to conclude that an NSP could be useful for promoting the spread of EC and the use of renewable energy sources for wastewater treatment to reduce the carbon footprint of the entire process.
\end{abstract}

Keywords: Electrocoagulation, iron electrode, COD, nanosecond pulse, specific energy consumption.

\section{FULL TEXT}


(C) 2020 The Authors. Published by ESG (www.electrochemsci.org). This article is an open access article distributed under the terms and conditions of the Creative Commons Attribution license (http://creativecommons.org/licenses/by/4.0/). 